\section{SAT0490 GENOME-WIDE DNA METHYLATION PROFILING OF OSTEOARTRHITIS PERIPHERAL BLOOD MONONUCLEAR CELLS REVEALS SLOWED EPIGENETIC AGING AMONG RAPID RADIOGRAPHIC PROGRESSORS: DATA FROM THE OSTEOARTHRITIS INITATIVE (OAI)}

A. Rivas ${ }^{1}$, M. Jeffries ${ }^{1,2} \cdot{ }^{1}$ Rheumatology, Immunology, and Allergy, University of Oklahoma Health Sciences Center; ${ }^{2}$ Arthritis \& Clinical Immunology Program, Oklahoma Medical Research Foundation, Oklahoma City, United States

Background: Extensive evidence has correlated epigenetic alterations in articular tissues with both the presence and progression of human osteoarthritis, but few analyses of blood cell epigenetic patterns have been done in OA.

Objectives: We examined the DNA methylation aging rate in peripheral blood mononuclear cells (PBMCs) at baseline from knee OA patients with rapid radiographic progression compared to well-matched nonprogressors enrolled in the Osteoarthritis Initiative (OAI).

Methods: PBMC DNA was obtained from baseline blood draws of 64 OA patients enrolled in the OAI longitudinal study. All patients had baseline symptomatic and radiographic $O A$. 32 rapidly-progressive $O A$ patients, defined as $\geq 1.0 \mathrm{~mm}$ radiographic joint space loss within 24 month follow-up were compared to 32 non-progressive patients. There were no differences in age, sex, race, BMI, baseline K/L grade, or calculated PBMC subset composition between rapid- and non-progressors. DNA methylation was quantified with Illumina HumanMethylation 450k arrays. Preprocessing was performed in GenomeStudio and normalized to internal controls. Epigenetic age was estimated with the algorithm described by Horvath et. al., using 353 age-associated CpG sites. This epigenetic age was compared to chronological age to calculate epigenetic-chronological age discordance $(\triangle \mathrm{Age})$ and group differences compared with a Student t-test. $\triangle$ Age was correlated with individual $\mathrm{CpG}$ methylation sites of rapid progressors, and Pearson values calculated. Correlation was considered significant if Pearson's $r$ values were $\leq-0.55$ or $\geq 0.55(p \leq 0.001)$. Pathway analysis of correlated genes was performed with the Ingenuity Pathway Analysis (IPA) system.

Results: The baseline DNA methylation aging rate in rapidly progressive (RP) knee OA patients was decelerated compared to nonprogressors (NP) and to chronological age ( $\triangle$ Age-RP: $-4.9 \pm 1.4$ vs. $\triangle$ Age-NP: $-0.071 \pm 1.3$ mean \pm SEM years less than chronological age, $\mathrm{p}=0.015)$. $1165 \mathrm{CpG}$ sites were correlated with $\triangle$ Age in rapid progressors, corresponding to 755 genes. Ontologic analysis of highly correlated genes showed association of the STAT3 pathway $(\mathrm{p}=6 \mathrm{E}-4)$, Notch signaling $(p=1 E-3)$, axonal guidance signaling $(p=7 E-3)$, CREB signaling $(p=2 E-2)$, NFAT signaling $(p=2 E-2)$, and autophagy $(p=4 E-2)$ among others. Associated upstream regulators included FGF2 ( $p=3 E-5)$, SMAD4 $(p=9 E-4)$, SMAD5 $(p=1 E-3$, TNF $(p=4 E-3)$, and TGFB1 $(p=4 E-3)$, among others Conclusions: Our data reveal that a decelerated peripheral blood differential DNA methylation age epigenotype is present at baseline in rapidly progressive knee OA patients, but not in nonprogressive knee OA patients. The genes correlated with this methylation age deceleration cluster in pathways previously associated with $\mathrm{OA}$ in articular tissues, suggesting that these pathways may systemically epigenetically dysregulated. Our data reinforce the notion that $O A$ is a heterogeneous disease composed of distinct subgroups, and suggests that future epigenetic investigation of immune cell subsets may be beneficial in unraveling $O A$ pathogenesis.

Acknowledgements: We gratefully acknowledge the assistance of Dr. Michael Nevitt, PhD, MPH, and Dr. John Lynch, PhD, of the University of California San Francisco and the OAI for their assistance in sample selection.

Disclosure of Interest: None declared

DOI: 10.1136/annrheumdis-2017-eular.6567

\section{SAT0491 USE OF HISTAMINE H1-RECEPTOR ANTAGONISTS IS ASSOCIATED WITH DECREASED PREVALENCE OF RADIOGRAPHIC KNEE OSTEOARTHRITIS: A CROSS- SECTIONAL ANALYSIS OF OSTEOARTHRITIS INITIATIVE DATA}

I. Shirinsky, V. Shirinsky. Institute of Fundamental and Clinical Immunology, Novosibirsk, Russian Federation

Background: Mast cells are prevalent in osteoarthritis (OA) synovial tissue and their presence is associated with structural damage [1]. H1-receptor antagonists block the action of histamine, which is the major mediator of mast cells, on specific receptors. Therefore, $\mathrm{H} 1$ receptor blockade may represent a way to prevent and treat OA. Currently, there is a lack of data investigating the effects of $\mathrm{H} 1$-receptor blockade on knee OA in humans.

Objectives: To evaluate cross-sectional association between the use of histamine $\mathrm{H} 1$-receptor antagonists and radiographic knee OA.

Methods: For the current analysis we used data from the publically available Osteoarthritis Initiative (OAI). We compared cross-sectionally the knees of OAI participants taking $\mathrm{H} 1$-receptor antagonists at baseline with knees of control participants. We used logistic regression models to assess the association between radiographic knee $\mathrm{OA}$ (defined as $\mathrm{KL}>2$ and $\mathrm{JSN}$ ) and the use of H1-receptor antagonists. Generalized estimating equations (GEE) were used to adjust for the correlation between knees. The models were adjusted for multiple covariates including age, race, and body mass index.

Results: A sample of 4377 OAl participants (8753 knees) was analyzed, 457 of them (914 knees) used histamine $\mathrm{H} 1$-receptors at baseline. The use of histamine
$\mathrm{H} 1$-receptor antagonists was associated with lower prevalence of radiographic knee $\mathrm{OA}$ in both crude (OR 0.77, $95 \mathrm{Cl} 0.64-0.91, \mathrm{p}<0.01)$ and adjusted (OR $0.79,95 \mathrm{Cl} 0.66-0.95, \mathrm{p}=0.01$ ) analyses.

Conclusions: Histamine $\mathrm{H} 1$-receptor antagonists are associated with decreased prevalence of knee OA. Further studies are warranted to determine whether $\mathrm{H} 1$-receptor blockade may be of benefit in the prevention and treatment of knee OA.

References:

[1] de Lange-Brokaar BJ, Kloppenburg M, Andersen SN, Dorjee AL, Yusuf E, Herb-van Toorn L, et al. Characterization of synovial mast cells in knee osteoarthritis: association with clinical parameters. Osteoarthritis and Cartilage 2016; 24: 664-671.

Disclosure of Interest: None declared

DOI: 10.1136/annrheumdis-2017-eular.2658

\section{SAT0492 INTRAMUSCULAR CORTICOSTEROID INJECTION VERSUS PLACEBO INJECTION IN HIP OSTEOARTHRITIS: A 12- WEEK BLINDED RANDOMIZED CONTROLLED TRIAL}

D. Dorleijn ${ }^{1}$, P. Luijsterburg ${ }^{1}$, M. Reijman ${ }^{2}$, M. Kloppenburg ${ }^{3}$, J. Verhaar ${ }^{2}$, P. Bindels ${ }^{1}$, K. Bos ${ }^{2}$, S. Bierma-Zeinstra ${ }^{1} .{ }^{1}$ General Practice; ${ }^{2}$ Orthopeadics, Erasmus MC, Rotterdam; ${ }^{3}$ Rheumatology, LUMC, Leiden, Netherlands

Background: Several international guidelines recommend intra-articular (IA) corticosteroid injections for patients with hip OA experiencing moderate to severe pain and no responding to oral analgesics. Previous research has shown a systemic effect of an intramuscular (IM) gluteal corticosteroid injection in patients with subacromial impingement shoulder pain. A clinically relevant effect of IM corticosteroid injections would offer a less complex, alternative treatment for patients' episodes of increased pain in hip OA.

Objectives: The trial aim was to assess the efficacy of an IM gluteal corticosteroid injection compared to a placebo injection on patients' reported hip pain severity in patients with hip OA, who were not responding on oral analgesics.

Methods: Patients in primary and secondary care were included if they met the clinical ACR and radiographic ( $K L$ score $>2)$ criteria for hip OA and scored a severity of hip pain $\geq 3$ on a scale of $0-10$ ( $0=$ no hip pain). Patients were randomized to receive either $40 \mathrm{mg}$ of triamcinolone acetate or saline (placebo) with an IA injection into the ipsilateral gluteus muscle. Primary outcome was severity of hip pain at 2 weeks, measured with numerical rating scale (NRS) in rest and during walking $(0-10 ; 0=$ no pain) and with the WOMAC pain subscale (0$100: 0=$ no pain). Secondary outcomes included hip pain severity (NRS, WOMAC pain, ICOAP), function (WOMAC function), stiffness (WOMAC stiffness), adverse events, and medical co-interventions at 2, 4, 6, and 12 weeks follow-up. Statistical analyses were performed based on the intention to treat principle. Linear mixed models with repeated measurements were used to analyze between group differences. The models were adjusted for variables that changed the effect estimate $>10 \%$.

Results: 107 of 422 screened patients were randomized. After informed consent, one randomized patient did not show up at the appointment for baseline measurement and subsequent injection and could, because of lack of data, not be included in the analyses. Finally, 52 patients in the corticosteroid injection group, and 54 in the placebo injection group were included in the analyses. $68 \%$ of the patients were female, and $25 \%$ were recruited by orthopedic surgeons. Mean age was 64 years (SD 11) and duration of OA was $\geq 1$ year for $70 \%$. At 2 weeks follow-up (table), the corticosteroid injection was statistically significant and clinically relevant associated with hip pain reduction at rest (coefficient -1.3 , $95 \% \mathrm{Cl}-2.3$ to -0.3 ) compared to placebo. The corticosteroid injection was also associated with significant hip pain reduction at 4, 6 and 12 weeks. Moreover, at almost all follow-up measurements the estimates showed significant differences in favor of the corticosteroid injection on WOMAC pain, function, stiffness and total score, and ICOAP. No significant differences between groups were found for adverse events and medical co-interventions.

Table. Results of the multivariable linear mixed model analyses with repeated measurements regarding primary outcomes between corticosteroid and placebo group.

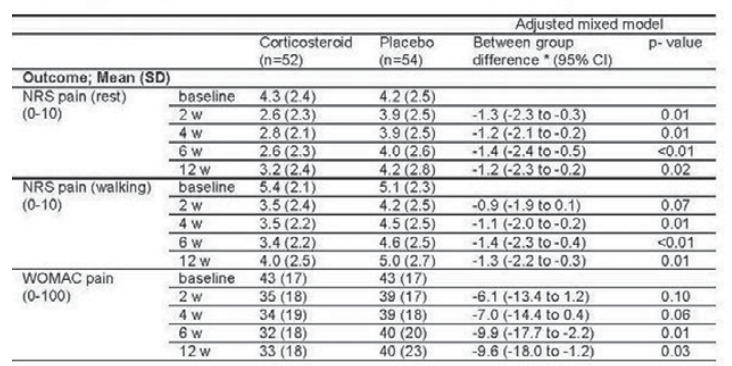

Models adjusted for baseline hip KL-score, ethnicity, hip stiffness, and patients expected effect; " placebo group is reference Index $(0=$ no pain): NRS = Numerical Rating Scale $(0=n O$ pain $):$ ICOAP = Intermittent and Constant OsteoArthritis Pain $(0=n$ Index) $w=$ weeks
pain

Conclusions: An IM gluteal corticosteroid injection was effective in hip pain reduction compared to placebo injection in patients with hip OA at 2 weeks 
folow-up. Moreover, the effect of the corticosteriod injection prolonged the entire 12 week follow-up period.

Disclosure of Interest: None declared

DOI: 10.1136/annrheumdis-2017-eular.4767

\section{SAT0493 MAINTAINING SUFFICIENT SERUM VITAMIN D LEVELS OVER TWO YEARS IS ASSOCIATED WITH IMPROVED KNEE STRUCTURAL AND SYMPTOMATIC OUTCOMES IN PEOPLE WITH KNEE OSTEOARTHRITIS: A POST HOC ANALYSIS OF THE VIDEO TRIAL}

S. Zheng ${ }^{1}$, X. Jin ${ }^{1}$, F. Cicuttini ${ }^{2}$, X. Wang ${ }^{1}$, Z. Zhu ${ }^{1}$, A. Wluka ${ }^{2}$, W. Han ${ }^{1}$ T. Winzenberg ${ }^{1}$, B.E. Antony ${ }^{1}$, D. Aitken ${ }^{1}$, L. Blizzard ${ }^{1}$, G. Jones ${ }^{1}$, C. Ding ${ }^{1}$. ${ }^{1}$ Menzies Institute for Medical Research, University of Tasmania, Hobart: ${ }^{2}$ Department of Epidemiology and Preventive Medicine, Monash University, Melbourne, Australia

Objectives: To describe whether maintaining sufficient serum vitamin $D$ levels in people with knee osteoarthritis $(\mathrm{OA})$ and baseline vitamin $\mathrm{D}$ insufficiency has an association with change in knee structures and symptoms over two years.

Methods: Participants ( $n=413$, age $63.2 ; 50 \%$ females) with symptomatic knee $\mathrm{OA}$ and vitamin $\mathrm{D}$ insufficiency were enrolled in a clinical trial. 340 participants $(82.3 \%)$ completed the study with 25-hydroxyvitamin D [25(OH)D] measurements at month 0,3 and 24 . Participants were classified as consistently insufficient (serum $25(\mathrm{OH}) \mathrm{D} \leq 50 \mathrm{nmol} / \mathrm{l}$ at month 3 and $24, \mathrm{n}=45$ ), fluctuating $(25(\mathrm{OH}) \mathrm{D}>50 \mathrm{nmol} / \mathrm{l}$ at either point, $\mathrm{n}=68)$ and consistently sufficient $(25(\mathrm{OH}) \mathrm{D}$ $>50 \mathrm{nmol} / \mathrm{l}$ at month 3 and $24, \mathrm{n}=226$ ) vitamin $\mathrm{D}$ groups. Knee cartilage volume, cartilage defects, bone marrow lesions (BMLs) and effusion-synovitis volume were assessed using MRI at baseline and month 24 . Knee symptoms were assessed at baseline, month 3,6 12 and 24 using Western Ontario and McMaster Universities Arthritis Index (WOMAC).

Results: The consistently sufficient group had significantly less loss of tibial cartilage volume ( $\beta: 2.1 \%, 95 \mathrm{Cl} \%: 0.3 \%, 3.9 \%)$, less increase in effusionsynovitis volume ( $\beta$ : $-2.5 \mathrm{ml}, 95 \mathrm{Cl} \%:-4.7,-0.2)$ and less loss of WOMAC physical function ( $\beta$ : $-94.2,95 \% \mathrm{Cl}:-183.8,-4.5)$ compared to the consistently insufficient group in multivariable analyses. In contrast, there were no significant differences in these outcomes between the fluctuating and consistently insufficient groups. Changes in tibiofemoral cartilage defects, BMLs and knee pain were similar between groups.

Table 1. Associations between maintaining sufficient 25- $(\mathrm{OH}) \mathrm{D}$ levels and changes in cartilage volume and effusion -volume over 24 months in patients with knee osteoarthritis

\begin{tabular}{lcc}
\hline & \multicolumn{2}{c}{ Multivariable analysis } \\
\cline { 2 - 3 } & $\beta(95 \% \mathrm{Cl})$ & $\mathrm{P}$ Value \\
\hline Total tibial Cartilage Volume Change $(\% / \mathrm{y})$ & \multicolumn{2}{c}{ Reference } \\
$\quad$ Consistently insufficient & $1.5(-0.5,3.5)$ & 0.15 \\
Fluctuating & $2.1(0.3,3.9)$ & $\mathbf{0 . 0 3}$ \\
Consistently sufficient & $\mathbf{0 . 0 2}$ \\
P for trend & \\
Effusion-Synovitis Absolute Volume Change (ml) $\quad$ Reference & \\
Consistently insufficient & $0.7(-2.5,3.9)$ & 0.66 \\
Fluctuating & $\mathbf{0 . 0 3}$ \\
Consistently sufficient & $-2.5(-4.7,-0.2)$ & $<\mathbf{0 . 0 1}$ \\
P for trend & & \\
\hline
\end{tabular}

Adjusted age, sex and BMI and change in season of blood sampling.

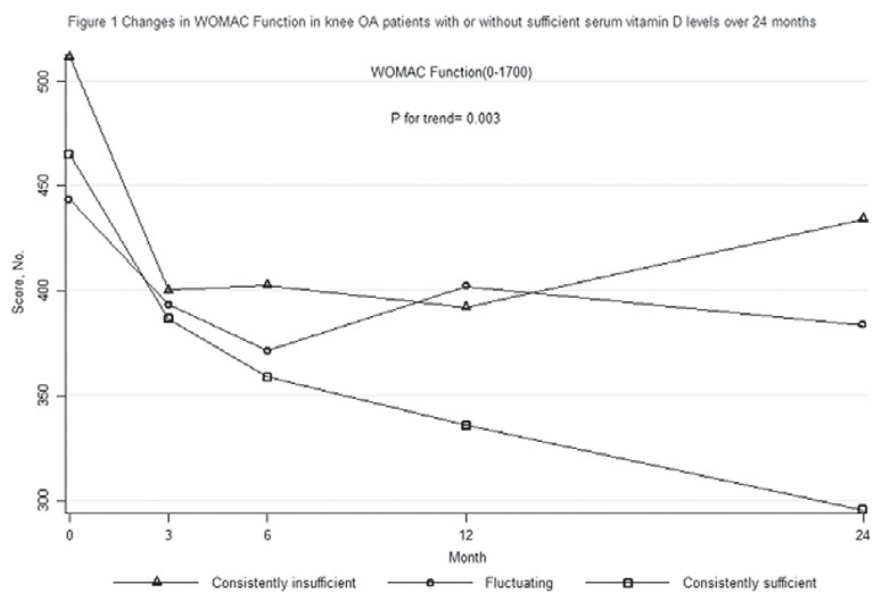

Conclusions: This post hoc analysis suggests beneficial effects of maintaining vitamin D sufficiency on cartilage loss, effusion-synovitis and physical function in people with symptomatic knee OA.

References:

[1] Cao, Y., et al., Vitamin D supplementation in the management of knee osteoarthritis: study protocol for a randomized controlled trial. Trials 2012;13:131. [2] Jin, X., et al., Effect of Vitamin D Supplementation on Tibial Cartilage Volume and Knee Pain Among Patients With Symptomatic Knee Osteoarthritis: A Randomized Clinical Trial. JAMA 2016; 315(10):1005-13.

Acknowledgements: We specially thank the participants who made this study possible, and we gratefully acknowledge the role of Vitamin D Effect on Osteoarthritis Study staff and volunteers in collecting the data. We thank the research assistants Jodi Barling, Kay Nguo, Judy Hankin and Alice Noone who were involved in the coordination of this study.

Disclosure of Interest: None declared

DOI: 10.1136/annrheumdis-2017-eular.1056

\section{SAT0494 EARLY TOLL-LIKE RECEPTOR 4 BLOCKADE IMPEDES THE BEHAVIOURAL AND HISTOLOGICAL CHARACTERISTICS OBSERVED IN A MIA-INDUCED ANIMAL MODEL OF OSTEOARTHRITIC PAIN}

M.M. Garcia ${ }^{1,2}$, D. Pascual ${ }^{1,2}$, E. Quesada ${ }^{2,3}$, J.A. Uranga ${ }^{4}$, C. Goicoechea ${ }^{2,5}$ ${ }^{1}$ Pharmacology, Universidad Rey Juan Carlos, Alcorcón; ${ }^{2}$ Unidad Asociada URJC-IQM-CSIC, Madrid-Alcorcón; ${ }^{3}$ Grupo Nucleósidos, Instituto química médica CSIC, Madrid; ${ }^{4}$ Histología, Universidad Rey Juan Carlos, Alcorcón; ${ }^{5}$ Pharmacology, Universidad Rey Juan Carlos, Madrid, Spain

Background: Contribution of Toll-Like Receptor 4 (TLR4) to pain sensitisation has been demonstrated to occur under chronic pain conditions. We previously described an antinociceptive effect of TLR4-A1, a TLR4 inhibitor, in two chronic pain conditions, peripheral neuropathic pain and osteoarthritis (OA).

Objectives: The aim of this study was to evaluate TLR4-A1 effect on allodynia and hyperalgesia in OA model, and to evaluate whether this effect is correlated with changes in spinal glial activation.

Methods: Wistar rats weighing $200-250 \mathrm{~g}$ were used. OA was induced by a single intraarticular injection of $2 \mathrm{mg}$ of monosodium iodoacetate (MIA) into the right knee joint of anaesthetised rats. TLR4-A1, $10 \mathrm{mgkg}^{-1}$, was intraperitoneally administered during the first five days post-MIA injection. TLR4A1 was synthesised by $\operatorname{Dr}$ Quesada. Vehicle-treatment (ethanol:saline, 1:9) was used as control. Each group was composed of 6 animals. After three weeks (day 22 post-MIA injection), animals were sacrificed for tissue collection. L3-L5 spinal segments were collected and embedded in paraffin wax. Eventually, samples were immune-stained with anti-GFAP or lba-1 antibodies. Photomicrographs were recorded to make montages of the entire spinal cord at a final magnification of $20 x$ ( $n=3$ per lumbar section). Total number of GFAP or lba-1 positive cells were counted separately in laminas I-II, III-IV and V-VI.

Results: Intraarticular injection of MIA increased microglial expression (lba-1 labelling) in the ipsilateral spinal cord compared to the contralateral side, being the difference statistically significant for the superficial $(\mathrm{I}-\mathrm{II},+72.25 \% ; \mathrm{P}<0.01)$ and deeper ( $\mathrm{V}-\mathrm{VI},+95.31 \% ; \mathrm{P}<0.001$ ) laminae of $\mathrm{L} 3$ and for the superficial laminae of $L 4(+87.5 \% ; P<0.01)$. In animals treated with TLR4-A1, Iba-1 labelling in the ipsilateral dorsal horn showed a similar pattern to the contralateral dorsal horn. Pre-treatment with TLR4 blocker prevented microglia activation after MIA-injection in L3 and L4 segments.

Intraarticular injection of MIA also increased the number of GFAP-positive activated astrocytes in the ipsilateral spinal cord compared to the contralateral side; in this case, statistically significant differences were found for the superficial $(\mathrm{I}-\mathrm{II} ;+41.62 \% ; \mathrm{p}<0.01)$ and middle (III-IV; $+64.35 \%, \mathrm{p}<0.001)$ laminae of L3 sections. GFAP in TLR4-A1-treated rats showed a similar pattern for the ipsiand the contra-lateral sides. That is, TLR4-A1 prevented L3 increased activated astroglia following MIA-injection.

Conclusions: Early toll-like receptor 4 blockade hampers spinal glial activation, which correlates with diminished allodynia and hyperalgesia observed in TLR4A1-treated animals in a model of MIA-induced OA. Although further studies are needed, TLR4 blockade could be a good option in the treatment of osteoarthritis. Acknowledgements: Franco $\mathrm{R}$ and Marquez A for technical support.

Granted: Ministerio de Economía y Competitividad. SAF2012-40075-C02-01.

Disclosure of Interest: None declared

DOI: 10.1136/annrheumdis-2017-eular.6022

\section{SAT0495 KNEE FUNCTION AND ENTHESITIS IN LONG STANDING OSTEOARTHRITIS, WHAT ULTRASOUND COULD TELL US?}

A. Abogamal, H. Aly, S. Ghanem, H. Saad on behalf of AL-Azhar MSUS school. Rheumatology, Alazhar Faculty of Medicine, Cairo, Egypt

Background: The enthesis around the knee including the quadriceps tendon and infrapatellar ligament insertions could be responsible for a significant cause of knee pain functional deterioration in long standing knee osteoarthritis.

Objectives: Ultrasound evaluation of the enthesis at the quadriceps tendon patellar insertion, infrapatellar ligament patellar and tibial insertions in patients with long standing knee osteoarthritis (KOA) and low knee function.

Methods: 410 Patients with KOA attending the outpatient rheumatology clinic of AL-Azhar university hospitals who had met the inclusion criteria:

- Primary Knee osteoarthritis.

- Kellegren - Lawrance scale grade III.

- At least 5 years disease duration.

Exclusion criteria:

- Patients with chronic diseases affects the patient function. 